\title{
Clinical relevance of Mycobacterium simiae in pulmonary samples
}

\author{
J. van Ingen*,\#, M.J. Boeree*, P.N.R. Dekhuijzen* and D. van Soolingen"
}

\section{ABSTRACT: The aim of the present study was to determine the clinical relevance of Mycobacterium simiae isolation from clinical samples.}

The medical files of patients in the Netherlands from whom M. simiae was isolated between 1999 and 2006 were reviewed in order to assess frequency and clinical relevance. Clinical relevance was defined as fulfilment of the diagnostic criteria of the American Thoracic Society.

From the files, 28 patients were identified, of whom six $(21 \%)$ met the American Thoracic Society diagnostic criteria. A slight (54\%) female predominance was observed, which is uncommon for nontuberculous mycobacteria isolation. Fulfilment of the diagnostic criteria and initiation of treatment were not in agreement; treatment results were poor.

Only a minority of clinical $M$. simiae isolates are clinically relevant and, applying the American Thoracic Society diagnostic criteria, the number of true infections is overestimated. Physicians in the Netherlands do not always use these criteria in daily practice, resulting in both over- and underdiagnosis of $M$. simiae infection. Further studies are required in order to improve diagnostic criteria and treatment regimens.

KEYWORDS: Infections in chronic obstructive pulmonary disease, infectious diseases, mycobacteria, mycobacteria other than tuberculosis, mycobacterial diseases, mycobacterium

n 1965, KARASSOVA et al. [1] reported the isolation of a new species of nontuberculous mycobacteria (NTM), which they named Mycobacterium simiae as it was isolated from rhesus monkeys (Latin simiae: of monkeys). In 1975, WEISZFEILER and KARCZAG [2] found that the previously described species $M$. habana was in reality also $M$. simiae.

Clinical $M$. simiae isolation at first seemed restricted to the southern USA, Israel and Cuba [3]. However, in due course, M. simiae isolation has been reported from many places in the world [4-6].

M. simiae is present in the environment and can contaminate medical equipment and laboratory samples $[3,7]$. Therefore, $M$. simiae isolation is not clinically relevant per se. Reports on $M$. simiae in the literature mainly describe single cases of infection; few authors have reported clinical relevance. The American Thoracic Society (ATS) has published guidelines for the diagnosis and treatment of NTM infections. Their diagnostic criteria are designed to distinguish between true and pseudo-infection, and thus to assess clinical relevance [8].

In the current study, the frequency and clinical relevance of $M$. simiae isolation in the
Netherlands were determined using the ATS diagnostic criteria available during the study period [8].

\section{METHODS}

The medical records of all patients in the Netherlands from whom $M$. simiae was isolated between January 1999 and January 2006 were examined. Demographic data, clinical data, drug susceptibility and whether the ATS diagnostic criteria available at the time [8] were met were recorded. The impact of the updated ATS diagnostic criteria published in 2007 was analysed separately [9].

The isolates of the patients were subjected to laboratory diagnosis by the Dutch National Institute of Public Health and the Environment (Bilthoven, the Netherlands). This is the national reference laboratory, providing identification, drug susceptibility testing and genotyping of Mycobacterium isolates for all health sector institutions in the Netherlands.

After ruling out membership of the $M$. tuberculosis complex using the Hain GenoType MTBC reverse line blot (Hain Lifescience, Nehren, Germany), an INNO-LiPA MYCOBACTERIA reverse line blot (Innogenetics, Ghent, Belgium) was used to identify common species of NTM,
AFFILIATIONS

*Dept of Pulmonary Diseases, Radboud University Nijmegen Medical Center, Nijmegen, and \# National Mycobacteria Reference Laboratory, National Institute for Public Health and the Environment, Bilthoven, the Netherlands.

CORRESPONDENCE

J. van Ingen

National Institute for Public Health and the Environment

Mycobacteria Reference Laboratory P.0. Box 1

3720 BA Bilthoven

The Netherlands

Fax: 31302744418

E-mail: jakko.van.ingen@rivm.nl

Received:

June 222007

Accepted after revision:

September 262007

STATEMENT OF INTEREST

None declared. 


\begin{tabular}{lccc} 
TABLE 1 & Baseline population characteristics \\
& \multicolumn{2}{c}{ ATS criteria } & Total \\
\cline { 2 - 3 } & Met & Not met & \\
\cline { 2 - 3 } & & & \\
\hline Subjects & 6 & 22 & $28(100)$ \\
Males & 2 & 11 & $13(46)$ \\
Females & 4 & 11 & $15(54)$ \\
Mean age yrs & $73^{\#}$ & 63 & 65 \\
Dutch origin & 6 & 20 & $26(93)$ \\
Pre-existing pulmonary disease & 6 & 20 & $26(93)$ \\
COPD & 5 & 16 & $21(75)$ \\
Lung cancer & 0 & 2 & $2(7)$ \\
Prior tuberculosis & 2 & 1 & $3(11)$ \\
Bronchiectasis & 2 & 3 & $5(18)$ \\
Current smoker & 0 & 3 & $3(11)$ \\
History of smoking & 3 & 11 & $14(50)$ \\
Alcohol abuse & 0 & 1 & $1(4)$ \\
HIV infection & 0 & 1 & $1(4)$ \\
Haematological malignancy & 0 & 1 & $1(4)$ \\
Otherwise impaired immunity & 0 & 2 & $2(7)$ \\
\hline
\end{tabular}

Data are presented as $\mathrm{n}$ or $\mathrm{n}(\%)$. ATS: American Thoracic Society; COPD: chronic obstructive pulmonary disease. ": methotrexate-associated pancytopenia and cystic fibrosis (each $n=1$ ). ${ }^{\#}$ : significant difference, $95 \%$ confidence interval 3.0-18.0; $p=0.008$.

including M. simiae. Prior to 2004, 16 S ribosomal DNA gene sequence analysis (151 bp) was performed after ruling out membership of the $M$. tuberculosis complex using the AccuProbe MTB DNA probe kit (GenProbe, San Diego, CA, USA).

Drug susceptibility testing was performed using the agar dilution method [10]. The drugs tested were isoniazid, rifampicin, ethambutol, streptomycin, cycloserine, protionamide, amikacin, ciprofloxacin, clofazimine, clarithromycin and rifabutin.

Pearson's Chi-squared test and Fisher's exact test were used for correlations. The study was approved by the Central
Committee on Research involving Human Subjects, ArnhemNijmegen regional office (Radboud University Nijmegen Medical Center, Nijmegen, the Netherlands).

\section{RESULTS}

M. simiae was isolated from 28 patients during the study period. No extrapulmonary isolates or disease were noted during the study period. The baseline patient characteristics are shown in table 1 . Six $(21 \%)$ patients met the ATS diagnostic criteria, based on features detailed in table 2 .

Patients who met the ATS diagnostic criteria for true NTM infection were significantly older than those who did not (73 versus $63 \mathrm{yrs}$; 95\% confidence interval (CI) of the difference 3.018.0; $\mathrm{p}=0.008$; table 1).

Productive cough (96\%), dyspnoea (54\%) and malaise (32\%) were the most commonly reported symptoms, although only patients who reported weight loss were significantly more likely to meet the ATS diagnostic criteria (odds ratio (OR) 9.0, 95\% CI 1.3-10.5; $\mathrm{p}=0.038$ ).

Chest radiography at the time of primary sampling mostly showed infiltrates $(54 \%)$, pre-existent emphysema $(25 \%)$ or fibrosis (25\%) and suspected malignant lesions (11\%). Of the chest radiographic abnormalities, both nodular $(p=0.040)$ and cavitary lesions $(p=0.040)$ were significantly associated with meeting the ATS diagnostic criteria.

The $M$. simiae isolates were cultured from sputum $(44 \%)$, bronchoalveolar lavage fluid (52\%) and lung biopsy specimens $(4 \%)$. Of all primary samples, $22 \%$ were positive for acid-fast bacilli on direct microscopy. An acid-fast bacillus-positive primary sample was significantly associated with meeting the ATS diagnostic criteria (OR 20.0, 95\% CI 1.7-30.9; $\mathrm{p}=0.010$ ).

Six patients started drug treatment; three of them had met the ATS diagnostic criteria. One of them was cured (as defined by improvement of symptoms and negative cultures until the end of the study period), one relapsed and one died due to progression of $M$. simiae disease (table 2). Three received treatment without having met the ATS diagnostic criteria, for a mean duration of 30 days (range 14-56 days), until an alternative diagnosis was made (disseminated Histoplasma

TABLE 2 Characteristics of patients with true Mycobacterium simiae infections

\begin{tabular}{|c|c|c|c|c|c|c|c|c|c|}
\hline Patient & Sex & Age yrs & Predisposing conditions & Symptoms & $\begin{array}{l}\text { Positive } \\
\text { cultures }\end{array}$ & $\begin{array}{l}\text { Chest } \\
\text { radiography }\end{array}$ & Histology & Treatment & Outcome \\
\hline 1 & $\mathrm{~F}$ & 79 & COPD & $\mathrm{PC}, \mathrm{Hp}, \mathrm{WL}, \mathrm{MA}$ & $2 / 2(\mathrm{Sm}+)$ & Infiltrate & - & 2ECipCla & Improved \\
\hline 2 & $\mathrm{~F}$ & 68 & TB & $\mathrm{PC}, \mathrm{Hp}$ & $4 / 4(\mathrm{Sm}+)$ & Cavities & $\mathrm{Gl}$ & 2HRZE6ECipCla & Relapse \\
\hline 3 & M & 75 & COPD & PC, Dysp, Fev, MA & $3 / 5$ (Sm-) & Infiltrate & - & None & Stable \\
\hline 4 & $\mathrm{~F}$ & 72 & COPD, bronchiectasis & PC, WL & $6 / 9(\mathrm{Sm}+)$ & Nodular & $N S I$ & None & Deteriorated \\
\hline 5 & M & 76 & COPD, bronchiectasis, TB & PC, Hp, Fev, WL, MA & $5 / 6(\mathrm{Sm}+)$ & Cavities, nodular & $\mathrm{Gl}$ & 1HRZ3RbECla & Relapse; died \\
\hline 6 & $\mathrm{~F}$ & 70 & COPD & PC, $\mathrm{Hp}, \mathrm{WL}$ & $3 / 3$ (Sm-) & Infiltrate & $\mathrm{NSI}$ & None & Stable \\
\hline
\end{tabular}

F: female; M: male; COPD: chronic obstructive pulmonary disease; TB: tuberculosis; PC: productive cough; Hp: haemoptysis; WL: weight loss; MA: malaise/asthenia; Dysp: dyspnoea; Fev: fever; Sm: smear; Gl: granulomatous inflammation; NSI: nonspecific inflammation; E: ethambutol; R: rifampicin; Rb: rifabutin; H: isoniazid; Z pyrazinamide; Cip: ciprofloxacin; Cla: clarithromycin. 
capsulatum infection and malignancy (each $\mathrm{n}=1)$ ) or identification of the NTM as M. simiae was communicated $(n=1)$.

The most commonly used drugs were rifampicin, ethambutol, ciprofloxacin and clarithromycin. Three patients met the ATS diagnostic criteria but did not receive treatment. In two, this decision was based on the estimated chance of successful treatment and the burden of treatment on the patients involved; in one, the stable clinical picture was the main reason. Two of the patients remained clinically stable and one showed radiological deterioration (table 2).

Drug susceptibility testing was performed for a single isolate of 25 patients, and invariably demonstrated in vitro resistance to rifampicin, ethambutol, isoniazid, streptomycin, amikacin and rifabutin. Resistance to ciprofloxacin (72\% of isolates) and clarithromycin (84\%) were frequent. Isolates were all susceptible to clofazimine and cycloserine, and most (76\%) were susceptible to protionamide.

The ATS has recently published new diagnostic criteria for NTM infections, with stricter radiological but less strict microbiological criteria for true infections. In addition, the criteria now state that "making the diagnosis of NTM lung disease does not, per se, necessitate the institution of therapy" [9]. Eight (29\%) patients in the present study met the new criteria, due to the less strict microbiological criteria.

\section{DISCUSSION}

In $21 \%$ of the 28 patients, $M$. simiae isolation represented clinically relevant disease, as defined by the ATS diagnostic criteria. This low degree of clinical relevance demonstrates the probable limited pathogenicity of $M$. simiae in humans.

In contrast to other NTM species, a small majority of the $M$. simiae isolates were cultured from samples from females. The low number of patients in the present study, however, prevents firm conclusions regarding sex distribution. Predisposing conditions, mainly pre-existent lung disease, were similar to those observed for other NTM species [8, 11]. During a nationwide survey in the USA, O'BRIEN et al. [11] recorded a similar degree of clinical relevance $(21 \%)$ and a comparable sex distribution ( $48 \%$ female) in 67 patients. The causes of this possibly unusual sex distribution remain unknown. Extrapulmonary infections are seldom reported and are restricted to immunocompromised hosts [5, 11]. Cases have occurred in the Netherlands before the present study period [12].

In those patients who met the ATS diagnostic criteria, treatment results were poor, with frequent relapses (table 2). Those who met the ATS diagnostic criteria but were left untreated remained clinically stable. Therefore, the benefit of current drug treatment regimens may be questioned, although the present study group is too small to draw firm conclusions. Alternatively, the ATS diagnostic criteria may not select the patients for whom treatment would be beneficial. Although designed for M. avium, M. kansasii and M. abscessus, the authors state that "there is no reason to believe these criteria would not be applicable to other species" [8]. The present data, however, suggest that the ATS criteria are not applicable to $M$. simiae since they overdiagnose true pulmonary $M$. simiae infections. Applying the latest ATS criteria [9], with more lenient microbiological criteria, further increased the number of true infections, since meeting the clinical and radiological criteria was common in the present study group; the microbiological criteria were the major hurdle.

Increasing the number of positive cultures required for diagnosis to more than three in $1 \mathrm{yr}$, or reservation of the diagnosis for histologically proven infections alone, may lead to improved selection of patients to benefit from treatment. In the present study, this would result in three cases $(11 \%$ of all patients) of true infection requiring treatment (patients 2, 4 and 5; table 2). In line with this observation, RYNKIEWICZ et al. [13], on the basis of stricter clinical and histological criteria, found $9 \%$ of 23 patients to have true $M$. simiae infections. Limited treatment results in the present study group demonstrate that the content and duration of treatment regimens require further study.

Although not suffering true infections, three patients in the present study were treated for NTM infection. This overtreatment may harm patients in terms of adverse effects and costs [14]. Moreover, it indicates the difficulty in diagnosing true infections. The limited exposure of physicians in the Netherlands to NTM disease leads to limited experience with the currently available, albeit imperfect, diagnostic criteria. This is probably one of the main reasons for the observed overand undertreatment.

When considering treating a patient, age and concomitant diseases might be a factor in the decision; the significantly higher age of patients who met the ATS criteria complicates this situation. The contribution of drug susceptibility testing results to the drug treatment decision remains limited, as the association between in vitro susceptibility and the in vivo response has not been established for most drugs [8]. Application of the new ATS criteria not only leads to diagnosis of more $M$. simiae infections, but also leaves the treatment decision more to the physician. For the infrequently encountered NTM, centralisation of expertise and easily accessible expert consultation is therefore important, as acknowledged in the new statement [9].

In conclusion, clinical Mycobacterium simiae isolation occurs infrequently in the Netherlands, with a slight female predominance uncommon to other nontuberculous mycobacteria. A minority of isolates represent true infection, which suggests limited pathogenicity in humans. The available diagnostic criteria are inadequate for the selection of patients for whom drug treatment for true Mycobacterium simiae infection would be beneficial. Critical evaluation of more patients from whom nontuberculous mycobacteria have been isolated will help to fine tune the criteria for improved diagnosis and treatment of nontuberculous mycobacteria infection.

\section{REFERENCES}

1 Karassova V, Weissfeiler J, Krasznay E. Occurrence of atypical mycobacteria in Macacus rhesus. Acta Microbiol Acad Sci Hung 1965; 12: 275-282.

2 Weiszfeiler J, Karczag E. Synonymy of Mycobacterium simiae, Karasseva et al., 1965 and Mycobacterium habana, Valdivia et al., 1971. Int J Syst Bacteriol 1976; 26: 474-477. 
3 Conger NG, O'Connell RJ, Laurel VL, et al. Mycobacterium simiae outbreak associated with a hospital water supply. Infect Control Hosp Epidemiol 2004; 25: 1050-1055.

4 Reller LB, Archibald LK, Jarvis WR, Grohskopf LA. Disseminated infection with simiae-avium group mycobacteria in persons with AIDS - Thailand and Malawi, 1997. JAMA 2002; 288: 157-158.

5 Sampaio JL, Pereira RM, Souza JR, Leite JP. Mycobacterium simiae infection in a patient with acquired immunodeficiency syndrome. Braz J Infect Dis 2001; 5: 352-355.

6 Legrand E, Devallois A, Horgen L, Rastogi N. A molecular epidemiological study of Mycobacterium simiae isolated from AIDS patients in Guadeloupe. J Clin Microbiol 2000; 38: 3080-3084.

7 Portaels F. Epidemiology of mycobacterial diseases. Clin Dermatol 1995; 13: 207-222.

8 Wallace RJ Jr, Glassroth J, Griffith DE, Olivier KN, Cook JL, Gordin F. Diagnosis and treatment of disease caused by nontuberculous mycobacteria. Am J Respir Crit Care Med 1997; 156: Suppl., S1-S25.

9 Griffith DE, Aksamit T, Brown-Elliot BA, et al. An official ATS/IDSA statement: diagnosis, treatment, and prevention of nontuberculous mycobacterial diseases. Am J Respir Crit Care Med 2007; 175: 367-416.

10 van Klingeren $B$, Dessens-Kroon $M$, van der Laan $T$, Kremer K, van Soolingen D. Drug susceptibility testing of Mycobacterium tuberculosis complex using a high throughput, reproducible, absolute concentration method. J Clin Microbiol 2007; 45: 2662-2668.

11 O'Brien RJ, Geiter LJ, Snider DE Jr. The epidemiology of nontuberculous mycobacterial diseases in the United States. Results from a national survey. Am Rev Respir Dis 1987; 135: 1007-1014.

12 Kuipers EJ, Hazenberg HJA, Ploeger B, Smit FW, de Jong A. Nontuberculous mycobacterial sternal osteomyelitis in a patient without predisposing condition. Neth J Med 1991; 38: 122-125.

13 Rynkiewicz DL, Cage GD, Butler WR, Ampel NM. Clinical and microbiological assessment of Mycobacterium simiae isolates from a single laboratory in southern Arizona. Clin Infect Dis 1998; 26: 625-630.

14 van Crevel R, de Lange WCM, Vanderpuye NA, et al. The impact of nontuberculous mycobacteria on management of presumed pulmonary tuberculosis. Infection 2001; 29: 59-63. 